Western University Scholarship@Western

$1-2010$

\title{
Bioenergy II: Characterization of the Pesticide Properties of Tobacco Bio-Oil
}

Christina J. Booker

Western University, cbooker2@uwo.ca

Rohan Bedmutha

Western University

Ian M. Scott

Kenneth Conn

Franco Berruti

Western University

See next page for additional authors

Follow this and additional works at: https://ir.lib.uwo.ca/ctlpub

\section{Citation of this paper:}

Booker, Christina J.; Bedmutha, Rohan; Scott, Ian M.; Conn, Kenneth; Berruti, Franco; Briens, Cedric; and Yeung, Ken K.-C., "Bioenergy II: Characterization of the Pesticide Properties of Tobacco Bio-Oil" (2010). Centre for Teaching and Learning Publications. 12.

https://ir.lib.uwo.ca/ctlpub/12 
Authors

Christina J. Booker, Rohan Bedmutha, Ian M. Scott, Kenneth Conn, Franco Berruti, Cedric Briens, and Ken K.-C. Yeung 


\title{
INTERNATIONAL JOURNAL OF CHEMICAL REACTOR ENGINEERING
}

\section{Bioenergy II: Characterization of the Pesticide Properties of Tobacco Bio-Oil}

\author{
Christina J. Booker* $\quad$ Rohan Bedmutha ${ }^{\dagger} \quad$ Ian M. Scott ${ }^{\ddagger}$ \\ Kenneth Conn ${ }^{* *} \quad$ Franco Berruti ${ }^{\dagger \dagger}$ \\ Cedric Briens $^{\ddagger \ddagger} \quad$ Ken K.-C. Yeung ${ }^{\S}$
}

${ }^{*}$ University of Western Ontario, cscorgie@uwo.ca

${ }^{\dagger}$ University of Western Ontario, rohan.bedmutha@gmail.com

${ }_{\ddagger}^{\ddagger}$ Agriculture and Agri-Food Canada, scotti@agr.gc.ca

**Agriculture and Agri-Food Canada, connk@agr.gc.ca

${ }^{\dagger \dagger}$ University of Western Ontario, berruti.ijcre@eng.uwo.ca

$\ddagger$ University of Western Ontario, cbriens@eng.uwo.ca

$\S$ University of Western Ontario, kyeung@uwo.ca

ISSN 1542-6580 


\title{
Bioenergy II: Characterization of the Pesticide Properties of Tobacco Bio-Oil*
}

\author{
Christina J. Booker, Rohan Bedmutha, Ian M. Scott, Kenneth Conn, Franco \\ Berruti, Cedric Briens, and Ken K.-C. Yeung
}

\begin{abstract}
Pyrolysis converts biomass such as agricultural and forestry waste into bio-oil, preserving some chemicals while creating other, new ones. Nicotine, a chemical present in tobacco leaves and a known pesticide, was found to remain intact during pyrolysis. As expected, insecticidal properties were observed for tobacco bio-oil. Pesticide characteristics of tobacco bio-oil have been observed on the Colorado potato beetle (CPB), a pest currently resistant to all major insecticides, as well as a few bacteria and fungi that do not currently respond well to chemical treatment. Unexpectedly, nicotine-free fractions of the bio-oil were also found to be highly lethal to the beetles and successful at inhibiting the growth of select microorganisms. Through GC-MS, it was found that the active, nicotine-free fractions were rich in phenolics, chemicals likely created from lignin during pyrolysis. While bio-oils in general are known to contain phenolic chemicals, such as cresols, to our best knowledge, quantitative analysis has not been performed to determine if these chemicals are solely responsible for the observed pesticide activities. Based on GC-MS results, ten of the most abundant chemicals, eight of which were phenolic chemicals, were identified and examined through bio-assays. A mixture of these chemicals at the concentration levels found in the bio-oil did not account for the bio-oil activity towards the microorganisms. Tobacco bio-oil may have potential as a pesticide, however, further analyses using liquid chromatography is necessary to identify the remaining active chemicals.
\end{abstract}

KEYWORDS: tobacco, bio-oil, pesticide, phenol, bacteria, fungi

* This work was supported by the Natural Sciences and Engineering Research Council of Canada (NSERC), by Agriculture and Agri-Food Canada through the funds provided to the Agricultural Biorefinery Innovation Network (ABIN), and by the University of Western Ontario. 


\section{Introduction}

Pyrolysis of biomass is currently being investigated around the world for applications of converting bulky, low-profit material into a valuable source of renewable energy and fine chemicals. Analysis of bio-oil has identified a complex mixture on the order of thousands of chemicals (Marsman, et al., 2008). The actual chemical composition of a bio-oil depends on the biomass source (Diebold, 2002) and pyrolysis conditions (Soltes and Elder, 1981), although many common chemicals are found in all bio-oils. Water, sugars, hydroxyaldehydes, hydroxyketones, carboxylic acids, and phenolics are the major groups of chemicals (Piskorz, et al., 1988).

Some of the recurring chemicals found in bio-oil, regardless of the biomass source, are known to be very toxic ( 50 to $500 \mathrm{mg} / \mathrm{kg}$ oral $\mathrm{LD}_{50}$ values). One example is phenol and its derivatives (Achladas, 1991, Garcia-Perez, et al., 2007, Luo, et al., 2004). Methyl substituted, monomeric phenolic compounds, ocresol, p-cresol, and m-cresol, have oral $\mathrm{LD}_{50}$ values of $121 \mathrm{mg} / \mathrm{kg}, 242 \mathrm{mg} / \mathrm{kg}$, and $207 \mathrm{mg} / \mathrm{kg}$, respectively, for rats (EI DuPont de Numours \& Co., 1983). Phenol itself has an oral $\mathrm{LD}_{50}$ value of $410-650 \mathrm{mg} / \mathrm{kg}$ for rats. The antifungal and antibacterial properties of cresols have been recognized for many years (Anonymous, 2006). In addition, cresols have been used as a wood preservative in the form of creosote. The origin of the phenolic compounds in bio-oil is attributed to the presence of lignin in the original biomass (Kleinert and Barth, 2008).

Recently, bio-oil from wood has been investigated for its wood preservative (Mourant, et al., 2007) properties against natural biodegradation and for its specifically anti-fungal properties (Mohan, et al., 2008, Mourant, et al., 2005). Phenolics were found to be present in the active bio-oil fractions (Mohan, et al., 2008, Mourant, et al., 2007). The antifungal activity was speculated to be due to the presence of phenolic compounds, although this conclusion was not supported by quantitative analysis. In addition to the antifungal properties of biooil, antibacterial and insecticidal properties of nicotine-free fractions of bio-oil from tobacco leaves have been discovered by our research group (Bedmutha, 2008, Booker, et al., 2008).

Bio-oil from tobacco plants is known to contain the highly toxic chemical nicotine (Scott D.S., 1997) (oral $\mathrm{LD}_{50}$ of $50 \mathrm{mg} / \mathrm{kg}$ for rats). In addition to its use in pharmaceuticals, nicotine is permitted as a pesticide in Canada, although its use as a pesticide is limited because of its extreme toxicity to and dermal absorption for mammals. Thus, it was expected that tobacco bio-oil would have pesticide properties due to the presence of nicotine. It was found in our research group, however, that nicotine-free fractions of tobacco bio-oil were lethal to the Colorado Potato Beetle (CPB, Leptinotarsa decemlineata), as well as successful at inhibiting the growth of two bacteria (Clavibacter michiganensis subsp. 
Michiganensis and Streptomyces scabies) and a fungus (Pythium ultimum) (Bedmutha, 2008, Booker, et al., 2008). The activity of the tobacco bio-oil towards the microorganisms will be discussed in this paper. S. scabies disfigures potato crops making them unmarketable, $C$. michiganensis seriously disfigures or kills tomato plants, and P. ultimum causes seedling damping off in a variety of vegetable plants. Tobacco bio-oil's pesticide properties are an exciting and potentially economically valuable finding.

Although the broad, general composition of bio-oil is known (mixture of alcohols, phenols, lignin, etc), no report, to our knowledge, has clearly identified which of these chemicals are responsible for the pesticide characteristics in biooil. Phenolic compounds have been assumed responsible for the observed antifungal activity in wood bio-oil, but this correlation has not been experimentally supported. While some of the identified chemicals in bio-oil are known to be active towards pests (ie. o-cresol, m-cresol, and p-cresol) (EI DuPont de Numours \& Co., 1983), the concentrations at which they are found in bio-oil may not be sufficient to account for the observed activity.

Thus, the purpose of this paper is to perform quantitative analysis to determine if the phenolic compounds present in tobacco bio-oil solely account for the pesticide activity towards the microorganisms S. scabies, C. michiganensis, and P. ultimum.

\section{Materials and Methods}

\section{Chemicals and Bio-oil}

Chemical standards 2,4-dimethyl phenol (97\%), 2,3-dimethyl phenol (99\%), 3,4dimethyl phenol (99\%), 4-ethyl phenol (99\%), o-cresol (99\%), p-cresol (99\%), mcresol (99\%), and (R)-limonene were obtained from Aldrich. Phenol and indole (99\%) were obtained from Mallinckrodt and Alfa Aesar, respectively. HPLC grade acetone was purchased from Fisher Scientific, while all other solvents (anhydrous ethyl ether, hexanes-200, dichloromethane, and methanol) were purchased from Caledon. Hydrochloric acid and solid sodium hydroxide were obtained from EM Science.

Tobacco leaves (dried at $60{ }^{\circ} \mathrm{C}$ ) were provided by Agriculture and AgriFood Canada, London, Ontario. The ground leaves used for bio-oil production had a Sauter mean diameter of $60 \mu \mathrm{m}$. Tobacco bio-oil was collected as the condensable vapours from an atmospheric fluidized bed reactor run at $450{ }^{\circ} \mathrm{C}$ under nitrogen. The vapour residence time was $5 \mathrm{~s}$. 


\section{Liquid-liquid fractionation}

The method used for tobacco bio-oil fractionation is illustrated in Fig.1. The tobacco bio-oil was dissolved in ether at a concentration of $375 \mathrm{mg} / \mathrm{mL}$ (Fraction $\mathrm{Z}$ ). This fraction was sterile filtered with a $2.5 \mathrm{~cm}$ diameter, $45 \mu \mathrm{m}$ pore size, nylon membrane syringe (Whatman, NJ, USA). A black residue remained on the walls of the vial and was dissolved in acetone and sterile-filtered (Fraction I). Fraction $\mathrm{Z}$ was separated into its aqueous (Fraction A) and organic components (Fraction B), using a water/ether extraction. Fraction B was further extracted by adding water and acidifying the water phase with concentrated $\mathrm{HCl}$ to $\mathrm{pH} 2$. It has been suggested that this acidification and neutralization step could alter the chemical composition of the resultant bio-oil fractions. However, this method was extremely successful at removing nicotine from other active chemicals, which was the goal of this step for this work. The ether fraction was collected as Fraction $\mathrm{C}$. The acidified water fraction was then neutralized with $\mathrm{NaOH}$ and ether was added, creating an ether fraction (Fraction D) and aqueous fraction (Fraction E). The ether in Fraction $\mathrm{C}$ was evaporated at room temperature, and a final separation created a methanol fraction (Fraction $\mathrm{M}$ ) and hexane fraction (Fraction H). Dilution factors were calculated for each fraction and the volume used in the bio-assays was appropriately adjusted to maintain the actual mass of the compounds between experiments. For further fractionations where Fraction $\mathrm{M}$ was the only consideration, the first two separations (creating Fraction B and Fraction $\mathrm{C}$ ), were performed at three times the dilution for better separation. The previous concentration of Fraction $\mathrm{M}$ was obtained through the evaporation step of Fraction C.

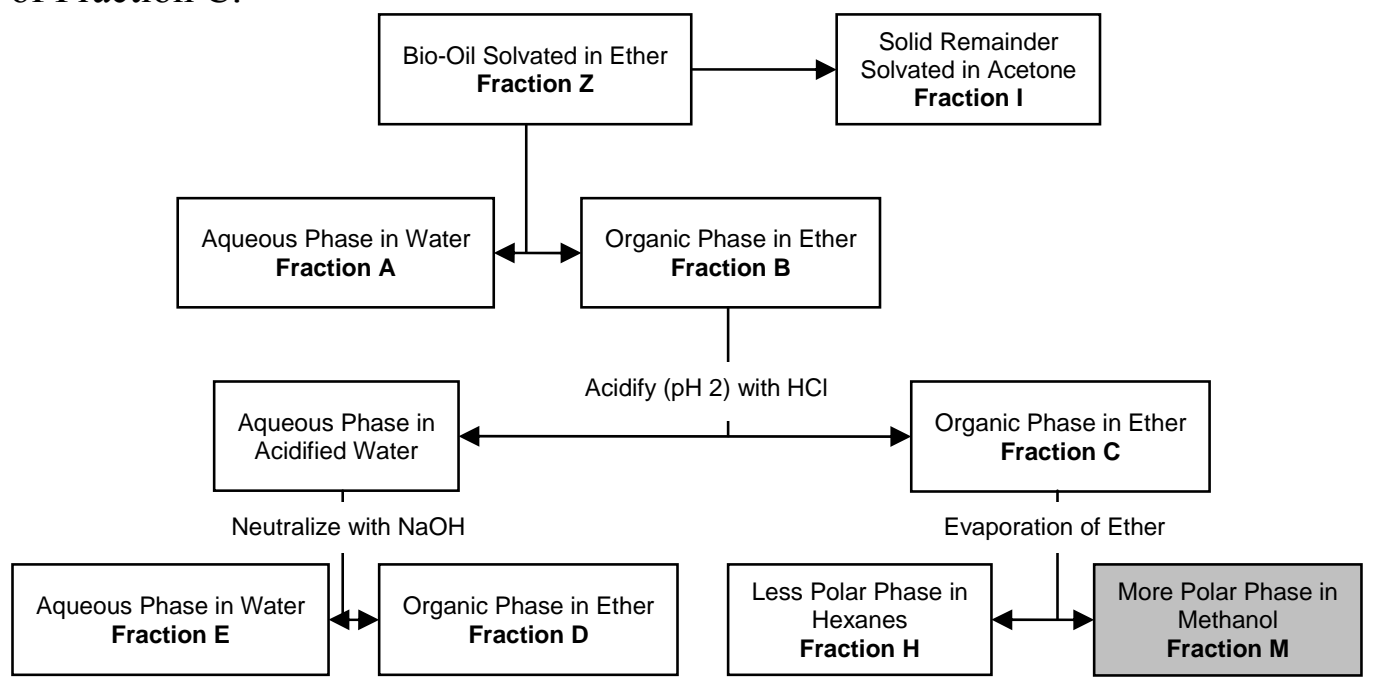

Figure 1. Fractionation scheme for tobacco bio-oil. Initial concentration for Fraction $\mathrm{Z}$ was 375 $\mathrm{mg} / \mathrm{mL}$. The shaded box indicates the fraction of interest in this paper, Fraction M. 


\section{Gas Chromatography-Mass Spectrometry}

A HP 6890 Series Gas Chromatography System with a Mass Selective Detector was used to analyze the bio-oil fractions. All experiments were performed on an HP-5MS, $30 \mathrm{~m}$ column obtained from Agilent Technologies with an i.d. of 0.25 $\mathrm{mm}$ and a film of $0.25 \mu \mathrm{m}$. The injector temperature and auxiliary temperature were maintained at $300{ }^{\circ} \mathrm{C}$. The oven temperature began at $60{ }^{\circ} \mathrm{C}$ for $2 \mathrm{~min}$, and then increased at $10{ }^{\circ} \mathrm{C} / \mathrm{min}$ to $280{ }^{\circ} \mathrm{C}$ and was held for $6 \mathrm{~min}$. A threshold of 150 was used, with a mass to charge scan range of 50 to 300 at a rate of 2.98 scans/s.

\section{Bio-assays for Microorganisms}

Streptomyces scabies (S. scabies), Pythium ultimum (P. ultimum), and Clavibacter michiganensis subsp. michiganensis (C. michiganensis) were obtained from Ken Conn, George Lazarovits, and Diane Cuppels, respectively, at the Southern Crop Protection and Food Research Centre, Agriculture and Agri-Food Canada, London Ontario. Streptomyces scabies, P. ultimum, and C. michiganensis were grown on yeast malt extract (YME), potato dextrose agar (PDA), and tryptic soy agar (TSA) media, respectively. Fresh bacterial cultures were maintained by streaking for isolated colonies and for the fungus by transferring an agar plug ( 5 $\mathrm{mm}$ diameter) from the actively growing edge of a culture. All cultures were incubated at $24{ }^{\circ} \mathrm{C}$ for up to 1 week before using them in the disc diffusion assay. The disc diffusion assay was used to test for antimicrobial activity of the bio-oil and standard solutions. Sterile $6 \mathrm{~mm}$ diameter filter paper discs were impregnated with $15 \mu \mathrm{L}$ of the sample or control solution, with the exception of the experiment displayed in Figure 2. Here, the volume of sample applied per disc was adjusted to account for the dilution factors during the fractionation steps (i.e., Fraction $\mathrm{Z}$ was more concentrated than Fraction M, and thus, less volume of Fraction $Z$ was applied to the disc). The discs were allowed to air-dry before being placed onto freshly inoculated plates. For the assay, bacteria from freshly grown plates were streaked onto new agar plates to cover the entire surface obtaining a lawn of growth. The discs were placed onto the inoculated plates and zones of no growth around the discs indicated inhibition. For the fungus, an agar plug was transferred from the actively growing edge of a colony to the centre of a new plate, and the discs placed about $1 \mathrm{~cm}$ away from the agar plug. Retarded growth of the fungus in the direction of the disc indicated inhibition. The plates were incubated at 24 ${ }^{\circ} \mathrm{C}$ for 3 days whereupon the results were recorded. The data reported is based upon triplicate inhibition measurements. Duplicate measurements were used when one of the triplicate experiments was invalid. 


\section{Results and Discussion}

\section{Pesticide Activity from the Bio-oil Fractions}

Pesticide activity in tobacco bio-oil was recently studied by our group (Bedmutha, 2008, Booker, et al., 2008). Disc diffusion agar experiments revealed growth inhibition for three of the fifteen species examined, namely S. scabies, $C$. michiganensis, and $P$. ultimum, by the naturally separated organic phase of tobacco bio-oil.

From this initial knowledge, the bio-oil was fractionated by liquid-liquid extraction to remove the nicotine and to simplify the chemical composition (Figure 1). The nicotine was removed from Fraction $\mathrm{B}$ by extraction with an acidic water phase, creating Fraction $\mathrm{C}$. This fraction contained no nicotine detectable by GC-MS. For this paper, Fraction $\mathrm{C}$ was further fractionated to separate the more polar, organic compounds, such as phenol (Fraction M) from the less polar, organic compounds, such as large alkanes (Fraction H). The inhibition data for the initial fraction, Fraction $\mathrm{Z}$, and the active, nicotine free, terminal fraction, Fraction $\mathrm{M}$, are included in Figure 2. Comparable activity was observed in both fractions. Thus, significant, active components were carried through the extraction process to Fraction $\mathrm{M}$.

The inhibition diameter measurements reported in this paper included the diameter of the $6 \mathrm{~mm}$ discs. Measurements were made to the nearest millimetre, so a measurement of $6 \mathrm{~mm}$ indicated only a slight region of inhibition around the disc, while measurements greater than this value showed increasing toxicity towards the species. 


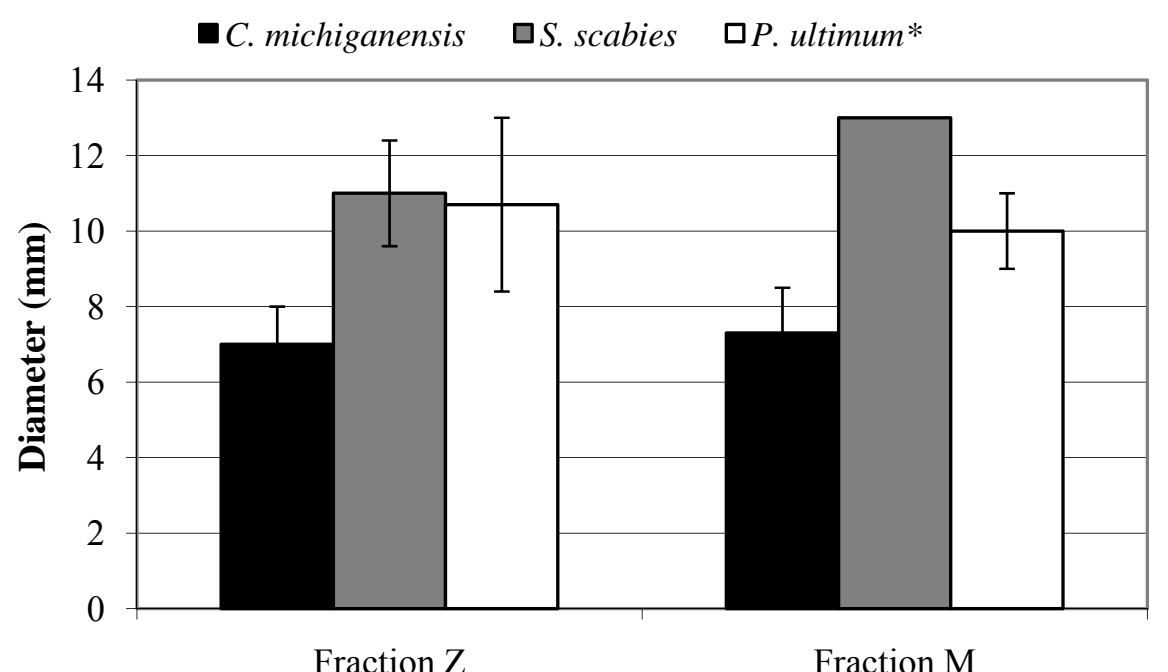

Figure 2. Diameter of inhibition for the initial fraction, Fraction $\mathrm{Z}$ and the active, terminal fraction, Fraction M. The fractionation scheme is shown in Figure 1. *Measurements for $P$. ultimum indicated regions of substantially less growth, but not complete inhibition. Sample standard deviation $(\sigma)$ values for replicates are shown as error bars $(+/-\sigma$, total length is $2 \sigma)$.

\section{Identification of Standards}

The Nist 2005 library was used to identify the peaks found in the GC-MS chromatogram of Fraction M (Figure 3). One hundred and seven peaks were detected and thirty-one chemicals were identified with qualities over $80 \%$. The largest peaks (peak area $>1.0 \%$ ), reflecting the most abundant chemicals present in Fraction M, were phenolic chemicals (Table 1). Six standards were obtained for the largest five peaks (one peak included both 3-methyl phenol and 4-methyl phenol). Being available in our inventory, four additional standards with relatively high peak areas were examined (peak area $\%>1.2 \%$ ). Thus, a total of ten standards were examined and their chemical structures are provided in Figure 3. 


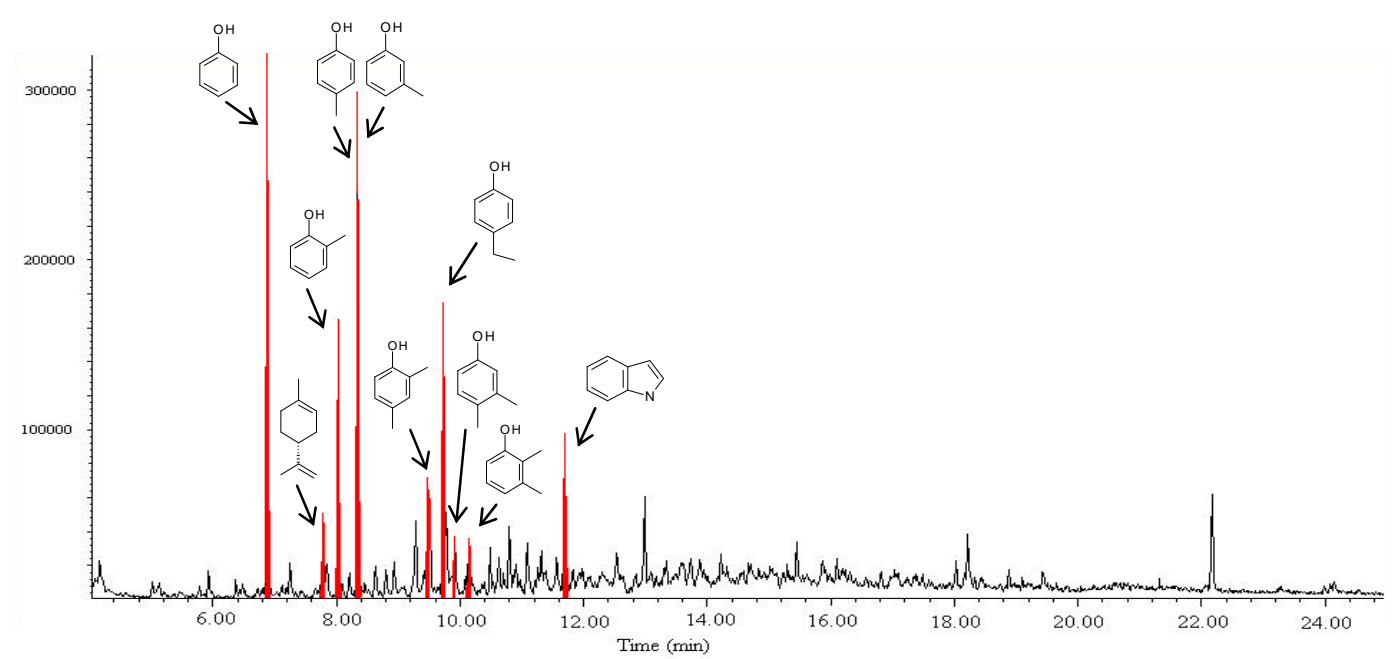

Figure 3: GC-MS chromatogram of Fraction $\mathrm{M}(50 \mu \mathrm{L}$ of Fraction $\mathrm{M}$ diluted to $1 \mathrm{~mL}$ in $\mathrm{MeOH})$. The peaks coloured in red and identified by chemical structures indicate the standards tested in this paper. For experimental conditions, see Methods section.

The concentrations of these standards in Fraction $\mathrm{M}$ were calculated using calibration curves (Table 1). Initially, to confirm the Nist 2005 identification, the standards were run individually and their retention times were compared to the peaks in Figure 3. After successful confirmation, these ten standards were run at 3 concentrations, ranging from $0.00625 \mathrm{mg} / \mathrm{mL}$ to $0.100 \mathrm{mg} / \mathrm{mL}$ such that the diluted sample peak fell between three standard calibration points. The integrated peak areas from these runs generated calibration curves with $\mathrm{R}^{2}$ values greater than 0.991. The slopes of the calibration curves for each standard were not the same, however. The slopes ranged from $1.00 \times 10^{8}$ area counts per concentration unit for 2,3-dimethyl phenol to $1.39 \times 10^{8}$ area counts per concentration unit for 4ethyl phenol. Thus, it was necessary that a calibration curve was generated for each standard as the peak area to concentration ratio was not constant between standards. These ten standards accounted for 54\% of the GC-MS chromatogram peak area of Fraction M, leaving the remaining 97 peaks to account for $46 \%$ of the peak area. Based upon the calculated concentration values, a mixture of these ten standards was tested on the three microorganisms. 
Table 1. The largest peaks (peak area $>1.0 \%$ ) identified from the GC-MS chromatogram of Fraction $\mathrm{M}$ are listed. The remaining 89 peaks $(<1.0 \%$ area each) are not included in this list. The Nist 2005 library was used to identify the peaks, and standards were used to confirm the identity of the indicated peaks. Chemicals confirmed by standards were used in the standard mixture at the indicated concentration. Concentration values were calculated using calibration curves.

\begin{tabular}{|c|c|c|c|c|c|}
\hline $\begin{array}{c}\text { Percent } \\
\text { Area } \\
(\%)\end{array}$ & $\begin{array}{l}\text { Retention } \\
\text { Time } \\
\text { (min) }\end{array}$ & $\begin{array}{l}\text { Chemical Name } \\
\text { (Identified by NIST 2005) }\end{array}$ & $\begin{array}{l}\text { Quality } \\
\text { (\%) }\end{array}$ & $\begin{array}{l}\text { Chemical Name } \\
\text { (Confirmed by } \\
\text { Standard) }\end{array}$ & $\begin{array}{c}\text { Concentration* } \\
\text { (mg/mL) }\end{array}$ \\
\hline 14.203 & 6.880 & Phenol & 91 & Phenol & 1.378 \\
\hline 14.064 & 8.344 & 3-methyl phenol & 96 & $\begin{array}{l}\text { 3-methyl phenol and } \\
\text { 4-methyl phenol }\end{array}$ & 1.340 \\
\hline 7.757 & 9.734 & 4-ethyl phenol & 95 & 4-ethyl phenol & 0.726 \\
\hline 6.631 & 8.025 & 2-methyl phenol & 98 & 2-methyl phenol & 0.646 \\
\hline 3.836 & 11.699 & indole & 95 & indole & 0.514 \\
\hline 2.790 & 22.173 & $\begin{array}{l}\text { cis-3-methyl-exo- } \\
\text { tricyclo[5.2.1.0(2.6)]decane }\end{array}$ & 58 & & \\
\hline 2.658 & 7.786 & d-limonene & 96 & d-limonene & 0.288 \\
\hline 2.480 & 9.483 & 2,4-dimethyl phenol & 96 & 2,4-dimethyl phenol & 0.273 \\
\hline 2.210 & 9.284 & 2-ethyl phenol & 95 & & \\
\hline 2.159 & 12.992 & 5-methyl-1H-phenol & 95 & & \\
\hline 2.083 & 9.785 & 3,5-dimethyl phenol & 96 & & \\
\hline 1.960 & 9.506 & 2,5-dimethyl phenol & 96 & & \\
\hline 1.787 & 18.215 & $\begin{array}{c}\text { 1-methoxy-3-(2- } \\
\text { hydroxyethyl)nonane }\end{array}$ & 58 & & \\
\hline 1.534 & 11.095 & 2-ethyl-6-methyl phenol & 60 & & \\
\hline 1.465 & 10.805 & 2-ethyl-5-methyl phenol & 93 & & \\
\hline 1.302 & 10.155 & 2,5-dimethyl phenol & 76 & 3,4-dimethyl phenol & 0.248 \\
\hline 1.243 & 9.916 & 2,3-dimethyl phenol & 96 & 2,3-dimethyl phenol & 0.120 \\
\hline 1.056 & 8.634 & 1H-indene, octahydro-, cis- & 58 & & \\
\hline
\end{tabular}

\section{Bio-assays of the Standard Compounds}

Surprisingly, this standard mixture was unsuccessful at inhibiting the growth of the three microorganisms. In contrast to the large region of inhibition observed for Fraction M, no region of inhibition was observed for the standard mixture. This result demonstrated that the cumulative action of the 10 chemicals accounting for $54 \%$ of the peak area was not responsible for the activity observed in Fraction M.

Past research shows that some of these standards are indeed active towards certain bacteria and fungi (Anonymous, 2006). Thus, before presuming that these ten standards had absolutely no impact on the activity of Fraction M, it was of 
interest to test these standards at a higher concentration. These standards were therefore tested individually at $100 \mathrm{mg} / \mathrm{mL}$, well over the calculated concentration of the standards in Fraction M (shown in Table 1). Nine of the ten standards were quite potent towards the three microorganisms (Figure 4). Only d-limonene was not active at this high concentration. The di-alkylated phenols (2,4-dimethyl phenol, 2,3-dimethyl phenol, 3,4-dimethyl phenol) were found to be more potent than the mono-alkylated phenols (2-methyl phenol, 3-methyl phenol, 4-methyl phenol). This was reflected by the larger regions of inhibition for the former compounds and the smaller regions of inhibition for the latter. However, the concentrations of the di-alkylated phenols $(0.120$ to $0.273 \mathrm{mg} / \mathrm{mL})$ in Fraction M were much lower than those of the mono-alkylated phenols $(0.646$ to 0.670 $\mathrm{mg} / \mathrm{mL}$ ). Based on the results in Figure 4, a cumulative toxic effect from the standards would be expected in the tobacco bio-oil.

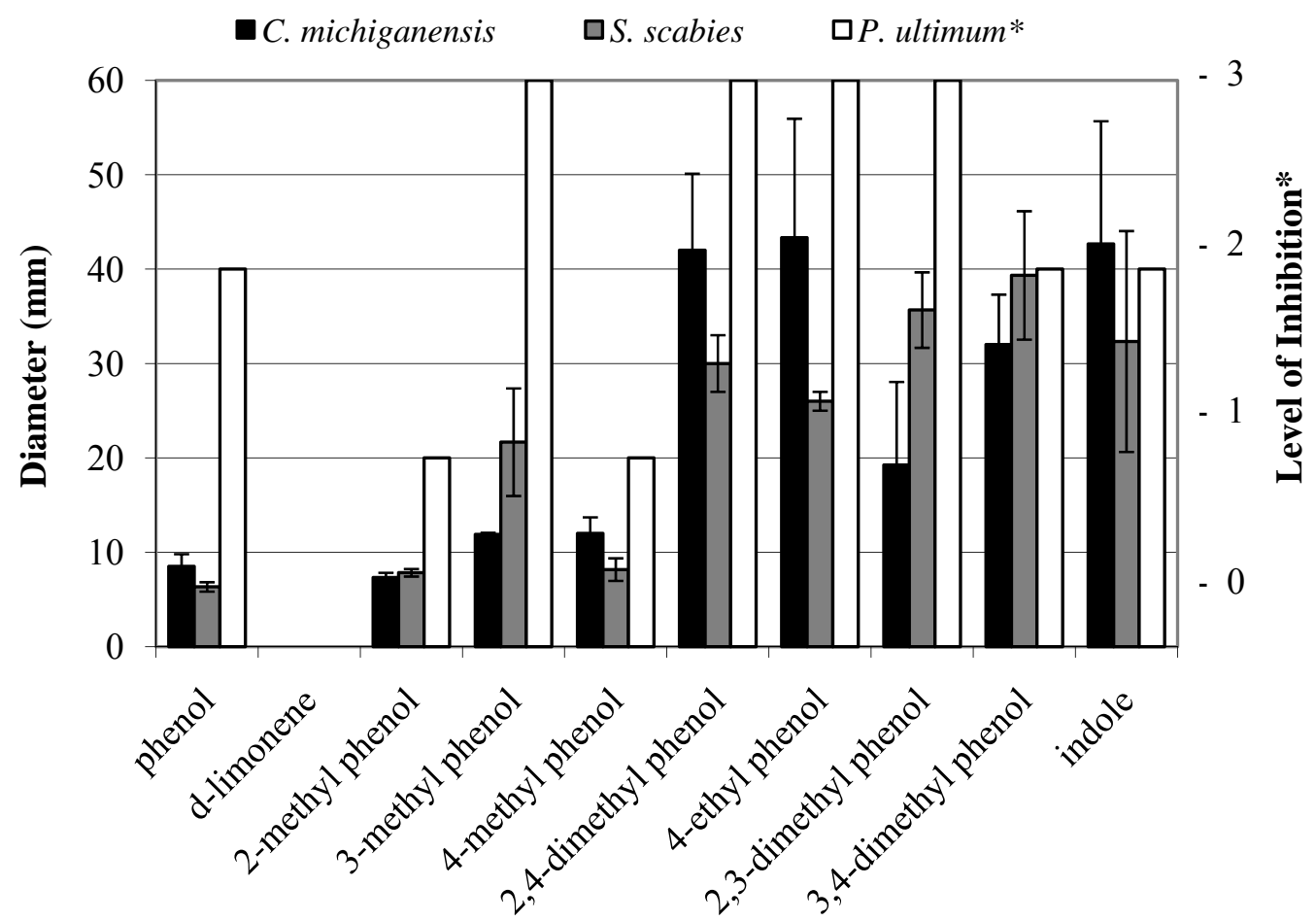

Figure 4. Diameter of inhibition for the bacteria C. michiganensis and S. scabies, and the level of inhibition for the fungus, P. ultimum. Sample standard deviation $(\sigma)$ values for replicates are shown as error bars $(+/-\sigma$, total length is $2 \sigma)$ for C. michiganensis and S. Scabies. *Diameter of inhibition measurements were not possible for this experiment with $P$. ultimum due to the extreme toxicity of the standards towards the fungus. Instead, a level system was used. The standard compounds were tested at $100 \mathrm{mg} / \mathrm{mL}$ in methanol.

*Level 0: complete growth

Level 1: thinner growth over entire plate
Level 2: thinner growth over part of plate

Level 3: no growth 
Although nine of the ten standards were toxic to the microorganisms at high concentration, the low concentration at which these standards were found in the bio-oil was not sufficient to account for the activity in Fraction M. However, it must be noted that not all of the phenolic and indole compounds in Fraction M were tested in this standard mixture. An additional ten other simple, alkylsubstituted phenolic compounds and one simple, alkyl-substituted indole compound were identified by the Nist 2005 library (quality $>80 \%$ ). These eleven compounds accounted for an additional $20 \%$ of the peak area. Therefore, to determine the extent to which the additional, related chemicals and the current, active standards contributed to the activity in Fraction M, the concentration of the standard mixture was increased.

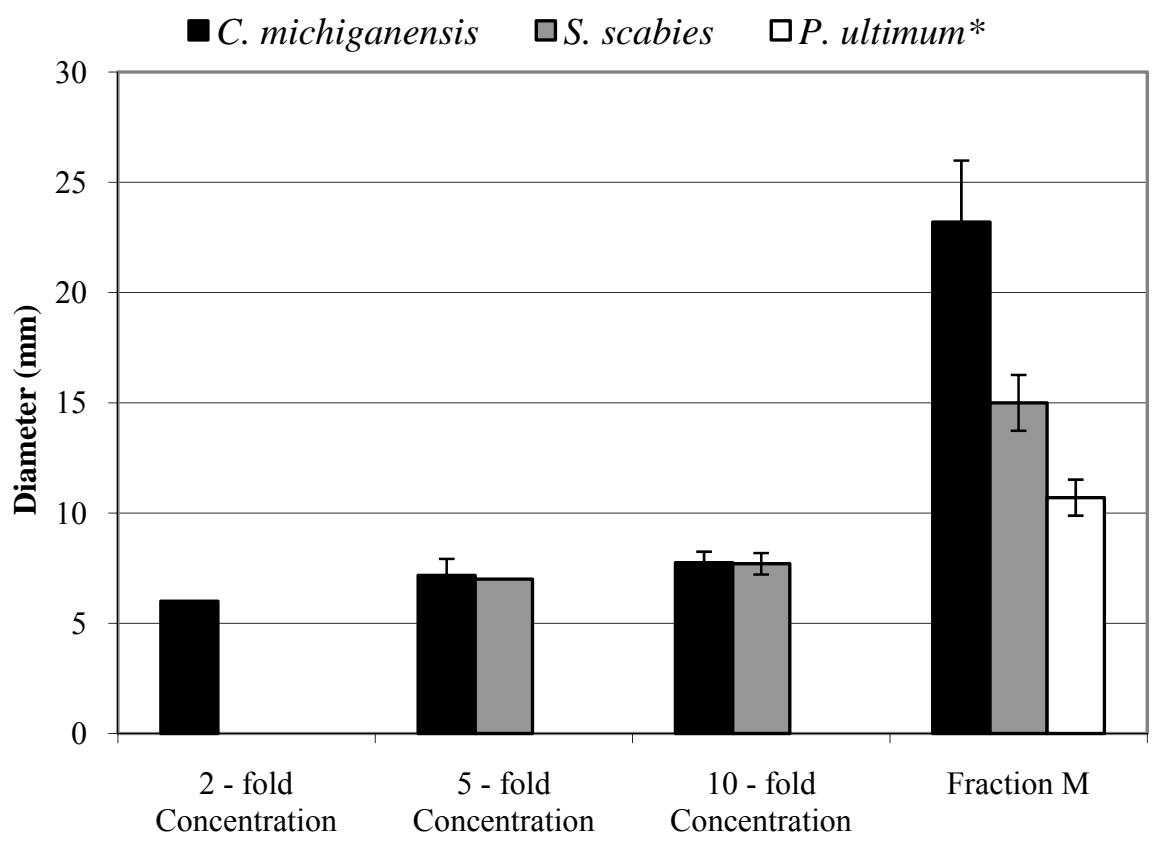

Figure 5: Diameter of inhibition for C. michiganensis, S. scabies, and P. ultimum. Discs were impregnated with the stated concentration of the standard mixture in methanol or with Fraction $\mathrm{M}$. *Measurements for P. ultimum indicated regions of substantially less growth, but not complete inhibition. Sample standard deviation $(\sigma)$ values for replicates are shown as error bars $(+/-\sigma$, total length is $2 \sigma$ ). 
Figure 5 displays the inhibition results of increasing the standard mixture concentration towards C. michiganensis, S. scabies, and P. ultimum. If the remaining eleven identified alkyl-substituted phenol and indole chemicals $(20 \%$ peak area) had similar potency to the ten obtained standards (54\% peak area), a two-fold increase in concentration of the standard mixture would more than account for these missing compounds. This, however, was not the case. At twofold concentration, only the smallest detectable activity was observed $(6 \mathrm{~mm}$ diameter of inhibition). Again, theoretically, if all the remaining chemicals $(46 \%$ peak area) had similar potency to the ten standards, increasing the standard mixture concentration two-fold would more than account for the activity in Fraction M. However, this was not found. Only the most minor inhibition was observed at 2-fold concentration.

An increase to five-fold concentration of the standard mixture showed minimal increase in activity. At ten-fold concentration the standard mixture was slightly more active than the two-fold concentration, but still not comparable to the larger regions of no growth around the discs impregnated with Fraction M. Therefore, the activity observed in Fraction $\mathrm{M}$ was not due to the tested standards, and was unlikely due to the remaining, eleven, simple phenol or indole compounds.

\section{Activity from Other Chemicals}

At ten-fold concentration, the standard mixture still did not match the inhibition levels of Fraction M. Thus, the ten standards accounted for less than $10 \%$ of the activity in the bio-oil. The origin of the majority of the activity has yet to be identified.

One option is that collectively, the chemicals comprising the remaining $46 \%$ peak area are responsible for the activity. This is highly unlikely as the remaining chemicals would need to account for over $90 \%$ of the activity. This would mean, on average, each remaining chemical would need to be 9 times more potent than the tested standards. Therefore, it is most probable that a few, extremely active chemicals have yet to be identified in Fraction M. These chemicals would need to be particularly potent, since the peak area for each remaining compound not tested in this paper was less than $2.8 \%$. Additionally, these potent chemicals are most likely not simple, alkyl-substituted phenolic or indole compounds.

It is possible that the potent chemicals are simply not detected by GC-MS, as only $40 \%$ of chemicals in bio-oil are GC-MS detectable (Meir, 1999). However, the fractionation scheme to create Fraction $\mathrm{M}$ seems to provide optimal conditions for selecting chemicals that would be detected by GC-MS. Chemicals in Fraction $\mathrm{M}$ are small (filtered through 0.45 um filter), polar enough to select 
the solvent methanol over the non-polar solvent hexane, but not polar enough to remain in water when an organic solvent is available (ether). The components in Fraction $\mathrm{M}$ are more likely to be detected by GC-MS over the other terminal fractions (Figure 1). However, if a new, potent chemical has been generated during pyrolysis of the tobacco bio-oil, its mass spectra would not be available in a library of chemicals. Thus, the mass spectra of low quality matches from the GC-MS of Fraction M should be analyzed manually.

Liquid chromatography with mass spectrometry detection is the next step in the analysis of the active bio-oil fraction. Gas chromatography requires that the compounds have a moderate boiling point (less than $280^{\circ} \mathrm{C}$ for our instrumental set-up). However, larger compounds in Fraction $\mathrm{M}$ likely have higher boiling points and thus do not reach the detector. In addition, the hard ionization technique (electron impact) used with our GC-MS method is poor in detecting larger molecules. Thus, liquid chromatography with a softer ionization detection technique will be very useful in analyzing Fraction $\mathrm{M}$ for active compounds. LCMS does not yet have a comprehensive database for identifying compounds, which makes this technique a more complex analysis tool compared to GC-MS. Although GC-MS was excellent at identifying phenolic compounds, it was found that these compounds are not the active pesticide chemicals. Thus, more involved techniques, such as LC-MS, will be used to examine the active bio-oil fractions in the future. Very potent chemicals remain to be identified in Fraction M.

\section{Conclusions}

Tobacco bio-oil contains nicotine, but nicotine is not responsible for the pesticide activity observed towards C. michiganensis, S. scabies, or P. ultimum. Liquidliquid extraction was used to generate bio-oil fractions. Activity assays identified an organic, nicotine-free fraction that was active towards the above species. According to the GC-MS data, this fraction was found to be rich in phenolic compounds, and standards were obtained for the largest peaks. A mixture was generated, accounting for $54 \%$ of the GC-MS peak area, but these chemicals did not account for the inhibition observed from Fraction $M$. These ten standard chemicals were responsible for less than $10 \%$ of the activity toward the microorganisms. Therefore, much to the surprise of the authors, the active chemicals in Fraction $M$ were not the alkyl substituted phenolic or indole chemicals tested in this paper.

Further tests are underway to identify the active chemicals in Fraction M. Using liquid chromatography, Fraction $\mathrm{M}$ will be separated into smaller fractions to identify the region of chemicals and eventually, the specific active chemicals. The identity of the active chemicals will reveal the potential for the use of tobacco bio-oil as a pesticide. 


\section{References}

"Final Report on the Safety Assessment of Sodium P-Chloro-M-Cresol, P-ChloroM-Cresol, Chlorothymol, Mixed Cresols, M-Cresol, O-Cresol, P-Cresol, Isopropyl Cresols, Thymol, O-Cymen-5-Ol, and Carvacrol", Int. J. Toxicol., 2006, 25, 29-127.

Achladas G. E., "Analysis of Biomass Pyrolysis Liquids - Separation and Characterization of Phenols", Journal of Chromatography, 1991, 542, 2, 263-275.

Bedmutha R., "Pesticides from Fast Pyrolysis of Agricultural and Forestry Residues", MSc Thesis, University of Western Ontario, London, 2008.

Booker C. J., Vogel T., Gloor A., Bedmutha R., Xu R., Ferrante L., Berruti F., Briens C., Yeung K. K.-C., "Characterization of Bio-Oils from Agricultural and Forestry Waste as Pesticides", 91 ${ }^{\text {st }}$ Canadian Chemistry Conference and Exhibition, Edmonton, AB, Canada, 2008, Presentation.

Diebold J. P., Bridgewater A., Ed. "Fast Pyrolysis of Biomass: A Handbook", 2002, CPL Press, Newbury, U.K., 243-292.

EI DuPont de Numours \& Co., "Cresols, Ortho-, Meta-, and Para-.", 1983, NTIS Report. No. OTS0205862.

Garcia-Perez M., Chaala A., Pakdel H., Kretschmer D., Roy C., "Characterization of Bio-Oils in Chemical Families", Biomass \& Bioenergy, 2007, 31, 4, 222-242.

Kleinert M., Barth T., "Phenols from Lignin", Chemical Engineering \& Technology, 2008, 31, 5, 736-745.

Luo Z. Y., Wang S., Liao Y. F., Zhou J. S., Gu Y. L., Cen K. F., "Research on Biomass Fast Pyrolysis for Liquid Fuel", Biomass \& Bioenergy, 2004, 26, 5, 455462.

Marsman J. H., Wildschut J., Evers P., de Koning S., Heeres H. J., "Identification and Classification of Components in Flash Pyrolysis Oil and Hydrodeoxygenated Oils by Two-Dimensional Gas Chromatography and Time-of-Flight Mass Spectrometry", J. Chromatogr. A, 2008, 1188, 1, 17-25.

Meir D., Bridgwater A., al e., Eds, "Fast Pyrolysis of Biomass: A Handbook", 1999, CPL Press, Newbury, UK, 92-101. 
Mohan D., Shi J., Nicholas D. D., Pittman C. U., Steele P. H., Cooper J. E., "Fungicidal Values of Bio-Oils and Their Lignin-Rich Fractions Obtained from Wood/Bark Fast Pyrolysis", Chemosphere, 2008, 71, 3, 456-465.

Mourant D., Riedl B., Rodrigue D., Yang D. Q., Roy C., "Phenol-FormaldehydePyrolytic Oil Resins for Wood Preservation: A Rheological Study", Journal of Applied Polymer Science, 2007, 106, 2, 1087-1094.

Mourant D., Yang D. Q., Lu X., Roy C., "Anti-Fungal Properties of the Pyroligneous Liquors from the Pyrolysis of Softwood Bark", Wood and Fiber Science, 2005, 37, 3, 542-548.

Piskorz J., Scott D. S., Radlein D., "Composition of Oils Obtained by Fast Pyrolysis of Different Woods", ACS Symposium Series, 1988, 376, 167-178.

Scott D.S. L. R. L., Piskorz J., Majerski P., Radlein D., Bridgewater A. V., Boocick D. G. B., Eds, "Developments in Thermochemical Biomass Conversion", 1997, Blackie Academic \& Prof.

Soltes E. J., Elder T. S., Goldstein I. S., Ed. "Organic Chemicals from Biomass", Chapter 5, 1981, CRC Press, Boca Raton, Florida, 63. 